\title{
Distance between configurations in MCMC simulations and the geometrical optimization of the tempering algorithms*
}

\author{
Masafumi Fukuma, ${ }^{a}$ Nobuyuki Matsumoto ${ }^{\dagger a}$ and Naoya Umeda ${ }^{b}$ \\ ${ }^{a}$ Department of Physics, Kyoto University \\ Kyoto 606-8502, Japan \\ ${ }^{b}$ PrincewaterhouseCoopers Aarata LLC \\ Otemachi Park Building, 1-1-1 Otemachi, Chiyoda-ku, Tokyo 100-0004, Japan \\ E-mail: fukumadgauge.scphys.kyoto-u.ac.jp, \\ hobu.meqauge.scphys.kyoto-u.ac.jp, naova.umeda1134dgmail.com
}

\begin{abstract}
For a given Markov chain Monte Carlo (MCMC) algorithm, we define the distance between configurations that quantifies the difficulty of transitions. This distance enables us to investigate MCMC algorithms in a geometrical way, and we investigate the geometry of the simulated tempering algorithm implemented for an extremely multimodal system with highly degenerate vacua. We show that the large scale geometry of the extended configuration space is given by an asymptotically anti-de Sitter metric, and argue in a simple, geometrical way that the tempering parameter should be best placed exponentially to acquire high acceptance rates for transitions in the extra dimension. We also discuss the geometrical optimization of the tempered Lefschetz thimble method, which is an algorithm towards solving the numerical sign problem.
\end{abstract}

37th International Symposium on Lattice Field Theory - Lattice2019

16-22 June 2019

Wuhan, China

\footnotetext{
*Report No.: KUNS-2778

${ }^{\dagger}$ Speaker.
} 


\section{Introduction}

In Markov chain Monte Carlo (MCMC) simulations, we often encounter a multimodal distribution, for which transitions between configurations around different modes are difficult. We introduced in [四] the distance between configurations to enumerate the difficulty of transitions.

In this talk, we mainly consider the simulated tempering algorithm implemented for an extremely multimodal system with highly degenerate vacua. Our distance enables us to investigate the algorithm in a geometrical way as follows. We first define a metric on the extended configuration space, and show that it is given by an asymptotically anti-de Sitter (AdS) metric [U, []]. We then show in a simple, geometrical way that the tempering parameter should be best placed exponentially to acquire high acceptance rates for transitions in the extra dimension. We further discuss the optimized form of the tempering parameter in the tempered Lefschetz thimble method (TLTM) [B], 田, 回], which is an algorithm towards solving the numerical sign problem. This talk is based on work [四, 口, 田].

\section{Definition of distance}

In this section, we briefly review the distance introduced in [四. Let $\mathscr{M} \equiv\{x\}$ be a configuration space, and $S(x)$ the action. Suppose that we are given an MCMC algorithm which generates a configuration $x$ from $y$ with the conditional probability $P(x \mid y)=(x|\hat{P}| y)$. We assume that it satisfies the detailed balance condition with respect to $p_{\text {eq }}(x) \equiv(1 / Z) e^{-S(x)}\left(Z \equiv \int d x e^{-S(x)}\right)$. We further assume that the Markov chain satisfies suitable ergodic properties so that $p_{\mathrm{eq}}(x)$ is the unique equilibrium distribution.

To define the distance, we consider the Markov chain in equilibrium. We denote by $\mathbb{W}_{n}$ the set of transition paths with $n$ steps in equilibrium, and by $\mathbb{W}_{n}(x, y)$, which is a subset of $\mathbb{W}_{n}$, the set of transition paths with $n$ steps which start from $y$ and end at $x$ in equilibrium. We define the connectivity between $x$ and $y$ by the fraction of the sizes of the two sets:

$$
f_{n}(x, y) \equiv \frac{\left|\mathbb{W}_{n}(x, y)\right|}{\left|\mathbb{W}_{n}\right|}=P_{n}(x \mid y) p_{\mathrm{eq}}(y)=f_{n}(y, x) .
$$

Here $P_{n}(x \mid y)=\left(x\left|\hat{P}^{n}\right| y\right)$ is an $n$-step transition matrix. We further introduce the normalized connectivity as

$$
F_{n}(x, y) \equiv \frac{f_{n}(x, y)}{\sqrt{f_{n}(x, x) f_{n}(y, y)}},
$$

with which we define the distance as follows:

$$
d_{n}(x, y) \equiv \sqrt{-2 \ln F_{n}(x, y)} .
$$

It can be shown that this distance gives a universal form at large scales for algorithms that generate local moves in the configuration space [四].

As an example, let us first consider the action $S(x)=(\beta / 2) \sum_{\mu=1}^{D} x_{\mu}^{2}$, which gives a Gaussian distribution in equilibrium. The distance can be calculated analytically for the Langevin algorithm:

$$
d_{n}(x, y)=\frac{\beta}{2 \sinh (\beta n \varepsilon)}|x-y|^{2},
$$


where $\varepsilon$ is the increment of the fictitious time. We thus find that a flat and translation invariant metric is obtained for Gaussian distributions.

As a second example, we consider the double-well action $S(x)=(\beta / 2)\left(x^{2}-1\right)^{2}$, which gives a multimodal equilibrium distribution. We again use the Langevin algorithm to calculate the distance. By making use of a quantum mechanical argument and an instanton calculation, we find that the distance behaves for $\beta \gg 1$ as

$$
\begin{aligned}
& d_{n}(x, y)=O\left(e^{-\beta n \varepsilon / 2}\right) \quad(\text { when } x, y \text { are around different modes }) \\
& \left.d_{n}(x, y) \propto \beta \quad \text { (when } x, y \text { are around the same mode }\right) .
\end{aligned}
$$

Therefore, we confirm that our distance quantifies the difficulty of transitions.

\section{Emergence of AdS geometry and the geometric optimization}

\subsection{Distance in the simulated tempering}

The simulated tempering [ $[$ ] is an algorithm to speed up the relaxation to equilibrium. In this algorithm, we choose a parameter $\beta$ in the action (e.g. an overall coefficient) as the tempering parameter, and extend the configuration space in the $\beta$ direction: $\mathscr{M} \rightarrow \mathscr{M} \times \mathscr{A}$, where $\mathscr{A} \equiv$ $\left\{\beta_{0}, \beta_{1}, \cdots, \beta_{A}\right\}=\left\{\beta_{a}\right\}_{a=0, \cdots, A}$ and $\beta_{0}$ is the original parameter of interest. We assume that $\left\{\beta_{a}\right\}$ are ordered as $\beta_{0}>\beta_{1} \cdots>\beta_{A}$. We set up a Markov chain in the extended configuration space $\mathscr{M} \times \mathscr{A}$ in such a way that the global equilibrium distribution becomes $P_{\mathrm{eq}}\left(x, \beta_{a}\right) \equiv w_{a} \exp \left[-S\left(x ; \beta_{a}\right)\right]$. We choose the weights $\left\{w_{a}\right\}$ to be $w_{a}=1 /(A+1) Z_{a}\left(Z_{a} \equiv \int d x e^{-S\left(x ; \beta_{a}\right)}\right)$. Expectation values are to be calculated by first realizing global equilibrium and then retrieving the subsample at $\beta_{a=0}$.

For multimodal systems, transitions between configurations around different modes are difficult. This situation can get improved by extending the configuration space as above because then such configuration can communicate easily by passing through the region with small $\beta_{a}$. The bene-

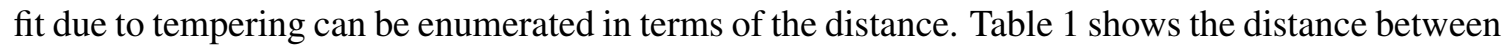
two modes in the original configuration space with and without tempering for the double-well action. We see that the introduction of the simulated tempering drastically reduces the distance.

\begin{tabular}{|c|c|c|}
\hline$n$ & without tempering & with tempering \\
\hline 10 & 39.1 & 26.5 \\
\hline 50 & 19.2 & 7.16 \\
\hline 100 & 16.9 & 4.35 \\
\hline 500 & 13.2 & 0.708 \\
\hline 1,000 & 11.7 & 0.106 \\
\hline 5,000 & 8.46 & $2.78 \times 10^{-8}$ \\
\hline
\end{tabular}

Table 1: Comparison of the distance with and without tempering [四].

\subsection{Emergence of AdS geometry}

We hereafter consider an extremely multimodal system with highly degenerate vacua. As a typical example, we use the action $S(x ; \beta) \equiv \beta \sum_{\mu=1}^{D}\left(1-\cos \left(2 \pi x_{\mu}\right)\right)$. 
According to the definition of our distance, $d_{n}(x, y)$ is negligibly small when $x$ and $y$ lie around the same mode, while $d_{n}(x, y)$ is large when $x$ and $y$ are around different modes. Therefore, when we investigate the large-scale geometry of $\mathscr{M}$, we can identify configurations around the same mode as a single configuration. We write the coarse-grained configuration space thus obtained as $\overline{\mathscr{M}}$ (for the cosine action, $\overline{\mathscr{M}}=\mathbb{Z}^{D}$ ). We can similarly coarse-grain the extended configuration space when the simulated tempering is implemented. We write the extended, coarse-grained configuration space as $\overline{\mathscr{M}} \times \mathscr{A}$.

We define the metric on $\overline{\mathscr{M}} \times \mathscr{A}=\left\{X \equiv\left(x, \beta_{a}\right)\right\}$ in terms of the distance:

$$
d s^{2}=g_{\mu v} d X^{\mu} d X^{v}=d_{n}^{2}(X, X+d X),
$$

where $X$ and $X+d X$ denotes nearby points in $\overline{\mathscr{M}} \times \mathscr{A}$. It can be shown that this metric is an asymptotically AdS metric [వ]. We here sketch the proof. We first note that the action is invariant under the lattice translation $x_{\mu} \rightarrow x_{\mu}+m(m \in \mathbb{Z})$ and thus, the metric components are independent of $x$. Furthermore, since the action is also invariant under the reflection $x_{\mu} \rightarrow-x_{\mu}$, there is no off-diagonal components. Thus we deduce that the metric takes the following form:

$$
d s^{2}=f(\beta) d \beta^{2}+g(\beta) \sum_{\mu=1}^{D} d x_{\mu}^{2} .
$$

We are left with determining two functions $f(\beta), g(\beta)$.

We first consider $g(\beta)$. Since transitions in the $x$ direction are difficult for larger $\beta, g(\beta)$ should be an increasing function of $\beta$ at least when $\beta$ is large. We here assume that the leading dependence on $\beta$ for $\beta \gg 1$ can be written as a power of $\beta$ :

$$
d_{n}^{2}((x, \beta),(x+d x, \beta))=\text { const. } \beta^{q} \sum_{\mu=1}^{D} d x_{\mu}^{2} \quad(\beta \gg 1),
$$

where $q$ is a constant. On the other hand, the functional form of $f(\beta)$ for $\beta \gg 1$ can be determined by evaluating the distance in the $\beta$ direction from the definition (2.3]) as follows. We first approximate the local equilibrium distribution in $\beta \gg 1$ by Gaussian. Then it turns out that the distance between two points $\left(x, \beta_{a}\right),\left(x, \beta_{a+1}\right)$ is a function of the ratio $\beta_{a} / \beta_{a+1}$ [ [d]. This means that the distance in the $\beta$ direction is invariant under scaling $\beta \rightarrow \lambda \beta$ for large $\beta$, and thus we obtain

$$
d_{n}^{2}((x, \beta),(x, \beta+d \beta))=\text { const. } \frac{d \beta^{2}}{\beta^{2}} \quad(\beta \gg 1) .
$$

Putting everything together, we conclude that the metric on $\overline{\mathscr{M}} \times \mathscr{A}$ is given by

$$
d s^{2}=l^{2}\left(\frac{d \beta^{2}}{\beta^{2}}+\alpha \beta^{q} \sum_{\mu=1}^{D} d x_{\mu}^{2}\right) \quad(\beta \gg 1)
$$

with constants $l, \alpha, q$. This is an AdS metric, as can be seen by the coordinate transformation $\beta \rightarrow(\sqrt{\alpha} q z / 2)^{-2 / q}$ :

$$
d s^{2}=\left(\frac{2 l}{q}\right)^{2} \cdot \frac{1}{z^{2}}\left(d z^{2}+\sum_{\mu=1}^{D} d x_{\mu}^{2}\right)
$$



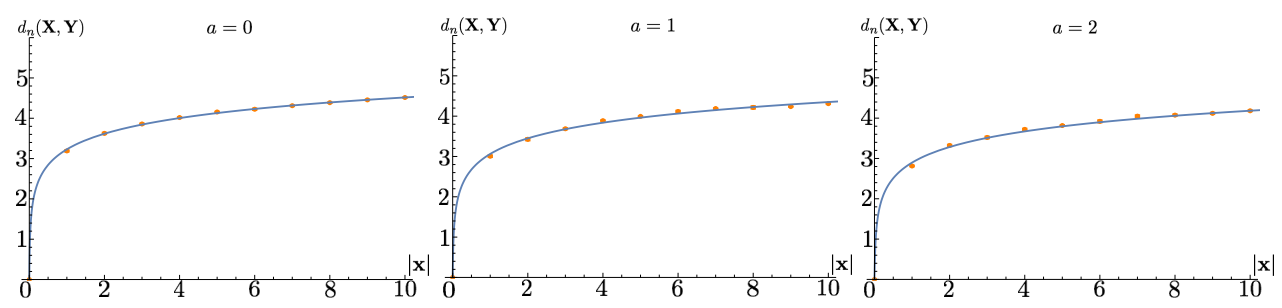

Figure 1: Calculated distances [0]. The solid line is the geodesic distance with the fitted parameters.

which is a Euclidean AdS metric in the Poincaré coordinates.

We can verify this metric in the following way. We first numerically calculate the distance $d_{n}\left(X \equiv\left(0, \beta_{a}\right), Y \equiv\left(x, \beta_{a}\right)\right)$ for $a=0,1,2$ and $x=1, \cdots, 10$. We then make a $\chi^{2}$ fit by using as the fitting function the geodesic distance calculated from the metric (B.5):

$$
\mathscr{I}\left(x, \beta_{a} ; l, \alpha, q\right) \equiv \frac{4 l}{q} \ln \left(\frac{\sqrt{(q \sqrt{\alpha}|x| / 4)^{2}+\beta_{a}^{-q}}+q \sqrt{\alpha}|x| / 4}{\beta_{a}^{-q / 2}}\right) .
$$

We carried out the above calculations for a two-dimensional $(D=2)$ configuration space, and obtained the results shown in Fig. $\mathrm{D}[\mathrm{⿴囗十}]$. The parameters are determined to be $l=0.0404(14)$, $\alpha=2.34(48) \times 10^{5}, q=0.289(12)$ with $\sqrt{\chi^{2} /(30-3)}=2.7$. The good agreement shows that the distances can be regarded as geodesic distances of an asymptotically Euclidean AdS metric.

\subsection{Geometrical optimization}

Our aim here is to optimize the functional form of $\beta_{a}=\beta(a)$ so that transitions in the extended direction become smooth. We make this optimization by referring to the geometry of the extended configuration space. Note that, since it is the parameter $a$ that is directly dealt with in MCMC simulations, we expect that the smooth transitions correspond to a flat metric in the extended direction when $a$ is used as one of the coordinates: $g_{\beta \beta} d \beta^{2}=$ const. $d a^{2}$. Since the geometry of $\overline{\mathscr{M}} \times \mathscr{A}$ is asymptotically $\operatorname{AdS}$ (B.5)), this means that $d \beta^{2} / \beta^{2} \propto d a^{2}$. This in turn determines the functional form of $\beta_{a}$ to be exponential in $a, \beta_{a}=\beta_{0} R^{-a}$ ( $\beta_{0}, R$ : constants).

We confirmed this expectation numerically by gradually changing the value of $\beta_{a}$ so that the distances between different modes are minimized [D]. The result is shown in Fig. \. We see that the optimized form of $\beta_{a}$ certainly takes an exponential form.

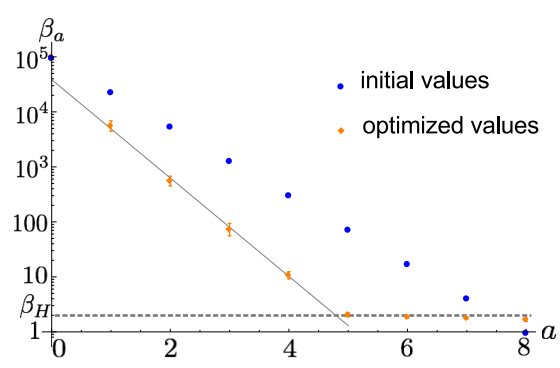

Figure 2: Optimized values for $\left\{\beta_{a}\right\}(a=1, \ldots, 8)$ [[]]. The blue dots are the initial values, and the orange dots are the resulting optimized values. 


\section{Tempering parameter in the tempered Lefschetz thimble method}

The tempered Lefschetz thimble method (TLTM) [3], 田, 回] (see also [9]) is an algorithm towards solving the sign problem. In this algorithm, by deforming the integration region from $\mathbb{R}^{N}$ to $\Sigma \subset$ $\mathbb{C}^{N}$, we reduce the oscillatory behavior of the reweighted integrals that appear in the following expression:

$$
\langle\mathscr{O}(x)\rangle=\frac{\int_{\Sigma} d z e^{-S(z)} \mathscr{O}(z)}{\int_{\Sigma} d z e^{-S(z)}}=\frac{\int_{\mathbb{R}^{N}} d x e^{-\operatorname{Re} S(x)} e^{-i \operatorname{Im} S(x)} \mathscr{O}(x) / \int_{\mathbb{R}^{N}} d x e^{-\operatorname{Re} S(x)}}{\int_{\mathbb{R}^{N}} d x e^{-\operatorname{Re} S(x)} e^{-i \operatorname{Im} S(x)} / \int_{\mathbb{R}^{N}} d x e^{-\operatorname{Re} S(x)}} .
$$

In Lefschetz thimble methods, such a deformation is made according to the antiholomorphic gradient flow: $\dot{z}_{t}^{i}=\left[\partial_{i} S\left(z_{t}\right)\right]^{*}$ with $z_{t=0}^{i}=x^{i}$, where the dot denotes the derivative with respect to $t$. This flow equation defines a map from $x \in \mathbb{R}^{N}$ to $z=z_{t}(x) \in \mathbb{C}^{N}$, and a new integration surface is given by $\Sigma_{t} \equiv z_{t}\left(\mathbb{R}^{N}\right) . \Sigma_{t}$ approaches a union of Lefschetz thimbles $\left\{\mathscr{J}_{\sigma}\right\}$ as $t \rightarrow \infty$, and the integrals remain unchanged under the continuous deformation thanks to Cauchy's theorem. Each thimble $\mathscr{J}_{\sigma}$ has a critical point $z_{\sigma}$ (where $\partial_{z^{i}} S\left(z_{\sigma}\right)=0$ ), and configurations $z \in \mathscr{J}_{\sigma}$ give the same phase, $\operatorname{Im} S(z)=\operatorname{Im} S\left(z_{\sigma}\right)=$ const. Thus, the oscillatory behavior of the reweighted integrals will get much reduced for large $t$. In the TLTM, we implement a tempering algorithm by choosing the flow time $t$ as the tempering parameter in order to cure the ergodicity problem caused by infinitely high potential barriers between different thimbles.

We can give a geometrical argument that the optimized form of flow times $t_{a}$ is linear in $a$ [团]. In fact, at large $t, \operatorname{Re} S\left(z_{t}(x)\right)$ increases exponentially as $\beta_{t} \propto e^{\text {const. } t}$. As in the simulated tempering, we expect that the optimal form of $\beta_{t_{a}}$ is an exponential function of $a$. Therefore, $t_{a}$ should be a linear function of $a$ (see also discussions in [8] $]$ ).

In the application of TLTM to the Hubbard model [四], we confirmed that this choice actually works well. Fig. [ 3 shows the acceptance rates between adjacent time slices, where $t_{a}$ is taken to be a piecewise linear function of $a$ with a single breakpoint. This choice results in the acceptance rates being roughly above 0.4 . Most notably, the acceptance rates become constant for larger $t$ (larger $a$ ), where $\Sigma_{t}$ gets close to the thimbles and the above discussion becomes more valid.

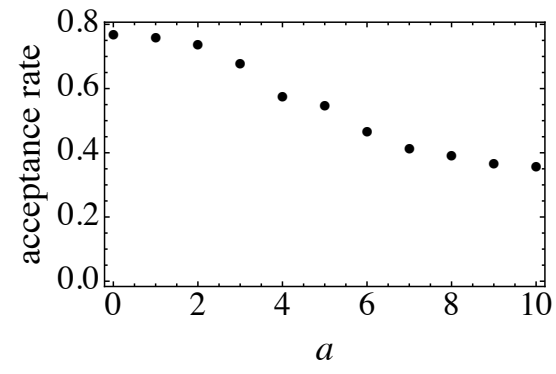

Figure 3: Acceptance rates in the $t_{a}$ direction with $\beta \mu=8$. Larger $a$ corresponds to larger $t_{a}$.

\section{Conclusion and outlook}

We introduced the distance between configurations, which quantifies the difficulty of transitions. We then discussed that an asymptotically AdS geometry emerges in the extended, coarsegrained configuration space, and showed that the optimization of the tempering parameter can be 
made in a simple, geometrical way. We further argued how to determine the optimized form of the tempering parameter in the tempered Lefschetz thimble method.

As for future work, it should be interesting to investigate the distance in the Yang-Mills theory, where the coarse-graining of the configuration space can be made by identifying configurations with the same topological charge as a single configuration. We further would like to apply the distance to models whose degrees of freedom can be interpreted as spacetime coordinates (e.g., matrix models) [ए0]. Then the geometry of the configuration space directly gives that of a spacetime. We expect that this formulation gives a systematic way to construct a spacetime geometry from randomness and provides us with a way to define a quantum theory of gravity.

A study along these lines is now in progress and will be reported elsewhere.

\section{Acknowledgments}

The authors greatly thank the organizers of LATTICE 2019. They also thank Andrei Alexandru, Hiroki Hoshina, Etsuko Itou, Yoshio Kikukawa, Yuto Mori and Akira Onishi for useful discussions. This work was partially supported by JSPS KAKENHI (Grant Numbers 16K05321, 18J22698 and 17J08709) and by SPIRITS 2019 of Kyoto University (PI: M.F.).

\section{References}

[1] M. Fukuma, N. Matsumoto and N. Umeda, Distance between configurations in Markov chain Monte Carlo simulations, JHEP 1712 (2017) 001 [1705 . 06097].

[2] M. Fukuma, N. Matsumoto and N. Umeda, Emergence of AdS geometry in the simulated tempering algorithm, JHEP 1811 (2018) 060 [1806.10915].

[3] M. Fukuma and N. Umeda, Parallel tempering algorithm for integration over Lefschetz thimbles, PTEP 2017 (2017) 073B01 [1703.00861].

[4] M. Fukuma, N. Matsumoto and N. Umeda, Applying the tempered Lefschetz thimble method to the Hubbard model away from half filling, Phys. Rev. D100 (2019) 114510 [1906. 04243 ].

[5] M. Fukuma, N. Matsumoto and N. Umeda, Implementation of the HMC algorithm on the tempered Lefschetz thimble method, in preparation.

[6] E. Marinari and G. Parisi, Simulated tempering: A New Monte Carlo scheme, Europhys. Lett. 19 (1992) 451 [hep-lat/9205018].

[7] A. Alexandru, G. Başar and P. Bedaque, Monte Carlo algorithm for simulating fermions on Lefschetz thimbles, Phys. Rev. D93 (2016) 014504 [1510 . 03258].

[8] A. Alexandru, G. Başar, P. F. Bedaque and N. C. Warrington, Tempered transitions between thimbles, Phys. Rev. D96 (2017) 034513 [1703.02414].

[9] M. Fukuma, N. Matsumoto and N. Umeda, Tempered Lefschetz thimble method and its application to the Hubbard model away from half filling, contributions in these proceedings.

[10] M. Fukuma and N. Matsumoto, in preparation. 